\title{
Significant effect of stacking on the electronic and optical properties of few-layer black phosphorus
}

\author{
Deniz Çakır, ${ }^{1, *}$ Cem Sevik, ${ }^{2, \dagger}$ and Francois M. Peeters ${ }^{1, \ddagger}$ \\ ${ }^{1}$ Department of Physics, University of Antwerp, Groenenborgerlaan 171, B-2020 Antwerpen, Belgium \\ ${ }^{2}$ Department of Mechanical Engineering, Faculty of Engineering, Anadolu University, Eskisehir TR 26555, Turkey
}

(Received 28 May 2015; revised manuscript received 8 September 2015; published 8 October 2015)

\begin{abstract}
The effect of the number of stacking layers and the type of stacking on the electronic and optical properties of bilayer and trilayer black phosphorus are investigated by using first-principles calculations within the framework of density functional theory. We find that inclusion of many-body effects (i.e., electron-electron and electron-hole interactions) modifies strongly both the electronic and optical properties of black phosphorus. While trilayer black phosphorus with a particular stacking type is found to be a metal by using semilocal functionals, it is predicted to have an electronic band gap of $0.82 \mathrm{eV}$ when many-body effects are taken into account within the $G_{0} W_{0}$ scheme. Though different stacking types result in similar energetics, the size of the band gap and the optical response of bilayer and trilayer phosphorene are very sensitive to the number of layers and the stacking type. Regardless of the number of layers and the type of stacking, bilayer and trilayer black phosphorus are direct band gap semiconductors whose band gaps vary within a range of $0.3 \mathrm{eV}$. Stacking arrangements that are different from the ground state structure in both bilayer and trilayer black phosphorus exhibit significant modified valence bands along the zigzag direction and result in larger hole effective masses. The optical gap of bilayer (trilayer) black phosphorus varies by $0.4(0.6) \mathrm{eV}$ when changing the stacking type. The calculated binding energy of the bound exciton hardly changes with the type of stacking and is found to be $0.44(0.30) \mathrm{eV}$ for bilayer (trilayer) phosphorous.
\end{abstract}

DOI: 10.1103/PhysRevB.92.165406

PACS number(s): 78.66.Db, 71.35.-y, 71.18.+y, 73.22.-f

\section{INTRODUCTION}

Triggered by the successful realization of a single layer of graphite (called graphene) [1,2], two-dimensional (2D) layered materials have drawn significant interest due to their potential use in the next generation of nanoelectronic and optoelectronic devices. The lack of a band gap in the electronic spectrum of graphene hampers its use in transistor applications, for instance [3-5]. Due to its atomically thin structure and the electronic properties with a direct band gap of $1.8 \mathrm{eV}, \mathrm{MoS}_{2}$ has been proposed as a promising candidate material [6] for nanoelectronics. Field effect transistors (FETs) based on a single layer of $\mathrm{MoS}_{2}$ was reported to have an ON/OFF ratio of $10^{8}$ and a carrier mobility of $200 \mathrm{~cm}^{2} / \mathrm{V} / \mathrm{s}$ [7], which may be further increased to $500 \mathrm{~cm}^{2} / \mathrm{V} / \mathrm{s}$ [8]. However, recent experiments have indicated that such a high carrier mobility in monolayer $\mathrm{MoS}_{2}$ may be just an overestimation due to the capacitive coupling between the gates of the devices [9].

Very recently, a new direct band gap semiconducting 2D material, called black phosphorus, has been successfully exfoliated [10-13] and introduced into the 2D crystal family. Besides its promising direct band gap, FETs based on black phosphorus have been reported to have a carrier mobility up to $1000 \mathrm{~cm}^{2} / \mathrm{V} / \mathrm{s}$ [10] and an ON/OFF ratio up to $10^{4}$ at room temperature [11], making it a promising candidate material for future technological applications. Single-layer $\mathrm{BP}$ has also been implemented into various electronic device applications including gas sensors [14], $p$ - $n$ junctions [15], and solar cells [16] due to its sizable band gap $(\sim 0.9 \mathrm{eV})$, and

\footnotetext{
*dcakir79@gmail.com

†csevik@anadolu.edu.tr

francois.peeters@uantwerpen.be
}

higher carrier mobility as compared to $\mathrm{MoS}_{2}$ [10,11,17-19], it is expected to be favorable for electronic device applications.

Although the electronic [16,20-25], thermal [26], and optical [27-32] properties of mono- and few-layer phosphorenes have been investigated previously, the role of the stacking type on its electronic and optical properties have remained an open question so far. In the present study we investigate how its electronic and optical properties change with respect to the number of stacking layers and the stacking type. The paper is organized as follows: Our computational approach is discussed in Sec. II. The structural and electronic properties of few-layer phosphorene is presented in Sec. III with special emphasis on the energy band gaps and effective electron and hole masses. The optical response is presented in Sec. IV together with exciton binding energy. Our results are summarized in Sec. V.

\section{COMPUTATIONAL METHOD}

We carry out first-principles calculations in the framework of density functional theory (DFT) as implemented in the Vienna $A b$ initio Simulation Package (VASP) [33,34]. The generalized gradient approximation (GGA) within the Perdew-Burke-Ernzerhof (PBE) [35] formalism and the HeydScuseria-Ernzerhof (HSE06) hybrid functional [36-38] is employed for the exchange-correlation potential. The projector augmented wave (PAW) method [39] and a plane-wave basis set with an energy cutoff of $400 \mathrm{eV}$ are used in the calculations. For geometry optimization the Brillouin-zone integration is performed using a regular $11 \times 15 \times 1 k$ mesh within the Monkhorst-Pack scheme [40]. The convergence criterion of the self-consistent field calculations is set to $10^{-5} \mathrm{eV}$ for the total energy. To prevent spurious interaction between isolated bilayers and trilayers, a vacuum spacing of at least $15 \AA$ is introduced. By using the conjugate gradient method, atomic positions and lattice constants are optimized until 
the Hellmann-Feynman forces are less than $0.01 \mathrm{eV} / \AA$ and pressure on the supercells is decreased to values less than $1 \mathrm{kB}$. Since bilayer and trilayer black phosphorus are layered materials, we take into account the van der Waals interaction between individual layers for the correct description of the structural properties of black phosphorus [41].

The weak screening and reduced dimensionality in 2D systems have a strong effect on their optical properties such that the excitonic effects have been shown to dominate the absorption spectra [42-45]. In such systems, many-body interactions (i.e., electron-electron and electron-hole interaction) must be taken into account for a correct description of the optical properties. In this work, we investigate the effect of the number of layers and the type of stacking on the optical spectra in bilayer and trilayer black phosphorus using the Bethe-Salpeter equation (BSE) as implemented in the VASP code $[46,47]$. In order to calculate the optical absorption spectrum and exciton binding energy, we follow the following steps. First, hybrid-DFT calculations are performed by using PBE-derived wave functions within the HSE06 approach. This is followed by one-shot $G W$ (i.e., $G_{0} W_{0}$ ) calculations to obtain the quasiparticle excitations [48-53]. Finally, we carry out BSE calculations on top of $G_{0} W_{0}$ in order to obtain the optical adsorption spectra by including excitonic effects using the Tamm-Dancoff approximation [54].

The BSE calculations are performed on a $9 \times 13 \times 1 k$ mesh within the Monkhorst-Pack scheme. The energy cutoff for the wave functions and for the response functions is set to $400 \mathrm{eV}$ and $200 \mathrm{eV}$, respectively. Since the number of empty bands significantly influences the relative position of the quasiparticle energy states, we use at least 100 empty bands. Within the current computational setup, the calculated quasiparticle gaps and exciton binding energies are then converged at least up to $0.1 \mathrm{eV}$. The four highest occupied valence bands and four lowest unoccupied conduction bands are included as a basis for the excitonic states. Since $G W$ calculations require a sufficiently large vacuum region, we use a vacuum region of at least $15 \AA$ to avoid spurious interaction between the periodic images. A complex shift of $\eta=0.1 \mathrm{eV}$ is employed to broaden the calculated absorption spectra.

\section{STRUCTURAL AND ELECTRONIC PROPERTIES}

In this study, three possible stacking types, illustrated in Figs. 1 and 2, are considered for both bilayer and trilayer black phosphorus. To realize these stacking types, individual monolayers were shifted with respect to the ground state structure. For instance, the top layer of $A B$ stacking in the bilayer is shifted by half of the cell along either the $a$ or $b$ direction in order to obtain AA stacking. The calculated structural parameters $(a$ and $b)$ summarized in Table I differ slightly for different stacking types. The most notable difference among the different stacking types is the interlayer distance between individual layers. It varies from $3.11 \AA$ in $\mathrm{AB}$ stacking to $3.70 \AA$ in $\mathrm{AC}$ stacking. In $\mathrm{AAB}$ stacking of the trilayer, we have two quite different interlayer distances, namely $3.11 \AA$ A between bottom and middle layers and $3.51 \AA$ between middle and top layers.
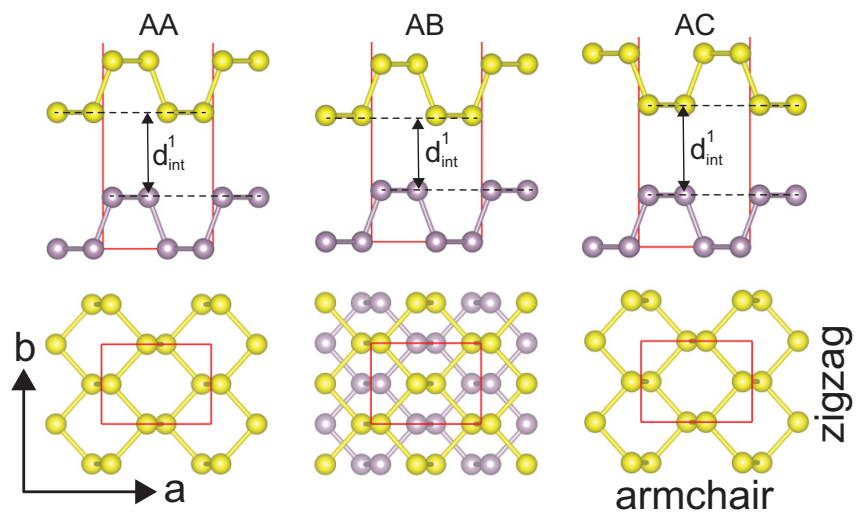

FIG. 1. (Color online) Side and top views of AA, AB, and AC stacked structures for bilayer black phosphorus. $d_{\text {int }}^{1}$ denotes the interlayer distance. The red rectangles in the bottom figures denote the unit cell. We also show the structural definition of the zigzag and armchair directions of black phosphorus.

To reveal the effect of the stacking type on the energy, we calculate the total energy difference $\Delta E$ (per atom) between a particular stacking type and the ground state structure. As is evident from Table I, while stacking types are quite different in both bilayer and trilayer systems, $\Delta E$ is found to be on the order of $20 \mathrm{meV} /$ atom, meaning that at finite temperatures (for instance at room temperature) one may have a high-energy structure, for instance AC stacking type in the bilayer and ACA stacking type in the trilayer case. This highlights the importance of studying the impact of the number of stacking layers and stacking type on the electronic and optical properties of black phosphorus.

Table I summarizes the electronic band gap $\left(E_{\text {gap }}\right)$ values calculated with PBE, HSE06, and $G_{0} W_{0}$ for bilayer and trilayer black phosphorus for different stacking types. As seen in Table I, using hybrid functionals significantly enlarges $E_{\text {gap }}$.
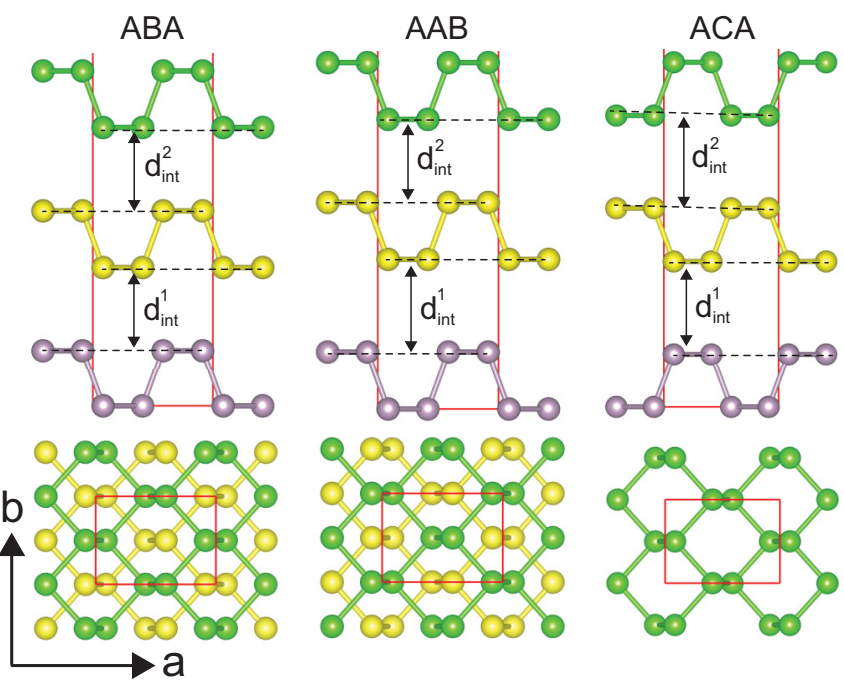

FIG. 2. (Color online) Side and top views of ABA, AAB, and ACA stacked structures for trilayer black phosphorus. $d_{\text {int }}^{1}$ and $d_{\text {int }}^{2}$ denote the interlayer distances. The red rectangles in the bottom figures denote the unit cell. 
TABLE I. Calculated interlayer distance $\left(d_{\text {int }}^{1}\right.$ and $\left.d_{\text {int }}^{2}\right)$, lattice parameters ( $a$ and $b$ ), electronic band gaps $\left(E_{\text {gap }}\right)$ for different exchangecorrelation functionals, optical gaps $\left(E_{\mathrm{opt}}\right)$, and exciton binding energies $\left(E_{\mathrm{bind}}=E_{\mathrm{gap}}^{G_{0} W_{0}}-E_{\mathrm{opt}}\right)$ for bilayer and trilayer black phosphorus for different stacking types. Distances and lattice parameters are given in units of $\AA$. Band gap values and energies are given in units of eV. $\Delta E$ is the total energy difference (in units of $\mathrm{meV} /$ atom) between a particular stacking type and the ground state structure calculated with the PBE functional including van der Waals correction. Here, the AB (ABA) stacking represents the ground state structure in bilayer (trilayer) black phosphorus. Electron $\left(m_{z}^{e}\right.$ and $\left.m_{a}^{e}\right)$ and hole $\left(m_{z}^{h}\right.$ and $\left.m_{a}^{h}\right)$ effective masses at the $\Gamma$ point along the zigzag $(z)$ and armchair $(a)$ directions are given in units of the free electron effective mass $\left(m_{0}\right)$. For comparison, we also include the results for single-layer (1L) black phosphorus.

\begin{tabular}{|c|c|c|c|c|c|c|c|c|c|c|c|c|c|c|}
\hline & $d_{\mathrm{int}}^{1}$ & $d_{\mathrm{int}}^{2}$ & $a$ & $b$ & $E_{\text {gap }}^{\mathrm{PBE}}$ & $E_{\text {gap }}^{\mathrm{HSE06}}$ & $E_{\text {gap }}^{G_{0} W_{0}}$ & $E_{\mathrm{opt}}$ & $E_{\text {bind }}$ & $\Delta E$ & $m_{z}^{h}$ & $m_{z}^{e}$ & $m_{a}^{h}$ & $m_{a}^{e}$ \\
\hline $1 \mathrm{~L}$ & & & 4.61 & 3.30 & 0.90 & 1.59 & 2.31 & 1.61 & 0.7 & & 6.33 & 1.24 & 0.13 & 0.14 \\
\hline AA & 3.50 & & 4.51 & 3.31 & 0.38 & 0.98 & 1.40 & 0.96 & 0.44 & 13.0 & 6.02 & 1.26 & 0.09 & 0.10 \\
\hline $\mathrm{AB}$ & 3.09 & & 4.51 & 3.31 & 0.42 & 1.03 & 1.45 & 1.02 & 0.43 & 0.0 & 2.70 & 1.32 & 0.11 & 0.11 \\
\hline $\mathrm{AC}$ & 3.71 & & 4.52 & 3.31 & 0.20 & 0.79 & 1.24 & 1.37 & 0.46 & 20.0 & 6.93 & 1.22 & 0.17 & 0.12 \\
\hline $\mathrm{ABA}$ & 3.13 & 3.13 & 4.50 & 3.32 & 0.21 & 0.80 & 1.10 & 0.81 & 0.29 & 0.0 & 1.55 & 1.29 & 0.08 & 0.09 \\
\hline $\mathrm{AAB}$ & 3.51 & 3.11 & 4.50 & 3.31 & 0.19 & 0.76 & 1.06 & 0.76 & 0.30 & 8.6 & 2.05 & 1.29 & 0.07 & 0.08 \\
\hline ACA & 3.68 & 3.69 & 4.53 & 3.30 & 0.00 & 0.49 & 0.82 & 1.34 & 0.33 & 27.0 & 6.39 & 1.10 & 0.19 & 0.15 \\
\hline
\end{tabular}

For instance, while $E_{\text {gap }}$ for bilayer black phosphorus with $\mathrm{AB}$ stacking is calculated to be $0.42 \mathrm{eV}$ with PBE-GGA, it becomes $1.03 \mathrm{eV}$ when using HSE06, consistent with previous calculations [16]. Figure 3 shows the band structures calculated using the HSE06 functional. Importantly, we note that the inclusion of many-body effects $\left(G_{0} W_{0}\right)$ further increases $E_{\text {gap }}$ of $\mathrm{AB}$ stacked bilayer phosphorene from $1.03 \mathrm{eV}$ to $1.45 \mathrm{eV}$ (see Table I). The ACA stacked trilayer is found to display metallic behavior when using the PBE functional. However, both HSE06 and $G_{0} W_{0}$ predict that it is a direct band gap semiconductor with an electronic band gap of 0.49 (0.82) $\mathrm{eV}$ with HSE06 $\left(G_{0} W_{0}\right)$. Therefore, to predict the electronic ground state accurately, hybrid and $G W$ calculations are essential. We observe that the electronic band gap of bilayer and trilayer black phosphorus can vary by $0.3 \mathrm{eV}$ when changing the stacking type, consistent with a recent work based on first-principles calculations [16]. For instance, $\mathrm{AA}, \mathrm{AB}$, and $\mathrm{AC}$ stacked bilayer black phosphorus have a quasiparticle gap of $1.40,1.45$, and $1.24 \mathrm{eV}$, respectively. Since the energy difference, $\Delta E$, between different stacking types is quite small, it is expected that mixed stacking in multilayer black phosphorus will be found in experiments. In addition, this competing energetics among different stacking types makes it easier to tune the electronic properties. Another important point is that, regardless of the number of layers and stacking type, bilayer and trilayer black phosphorus has a direct band gap at the $\Gamma$ point.

Black phosphorus has a highly anisotropic band structure around the band gap at the $\Gamma$ point. Both valence and conduction bands have a much more significant band dispersion along the armchair direction as compared to the zigzag direction. Table I summarizes hole and electron effective masses along the zigzag and the armchair directions at the $\Gamma$ point. The effective mass is calculated from the band structure using the following formula for the mass tensor,

$$
m_{i, j}^{*}=\hbar^{2}\left(\frac{\partial^{2} E}{\partial k_{i} \partial k_{j}}\right)^{-1}
$$

where $\hbar$ is the reduced Planck constant, $k_{i}\left(k_{j}\right)$ is the wave vector along the $i(j)$ direction, and $E$ is the energy eigenvalue. Here, the $i$ and $j$ directions are used for the zigzag $(z)$ and armchair ( $a$ ) directions of black phosphorus. We present results for the diagonal components of the mass tensor in Table I. For a reasonable numerical estimation of the second derivatives

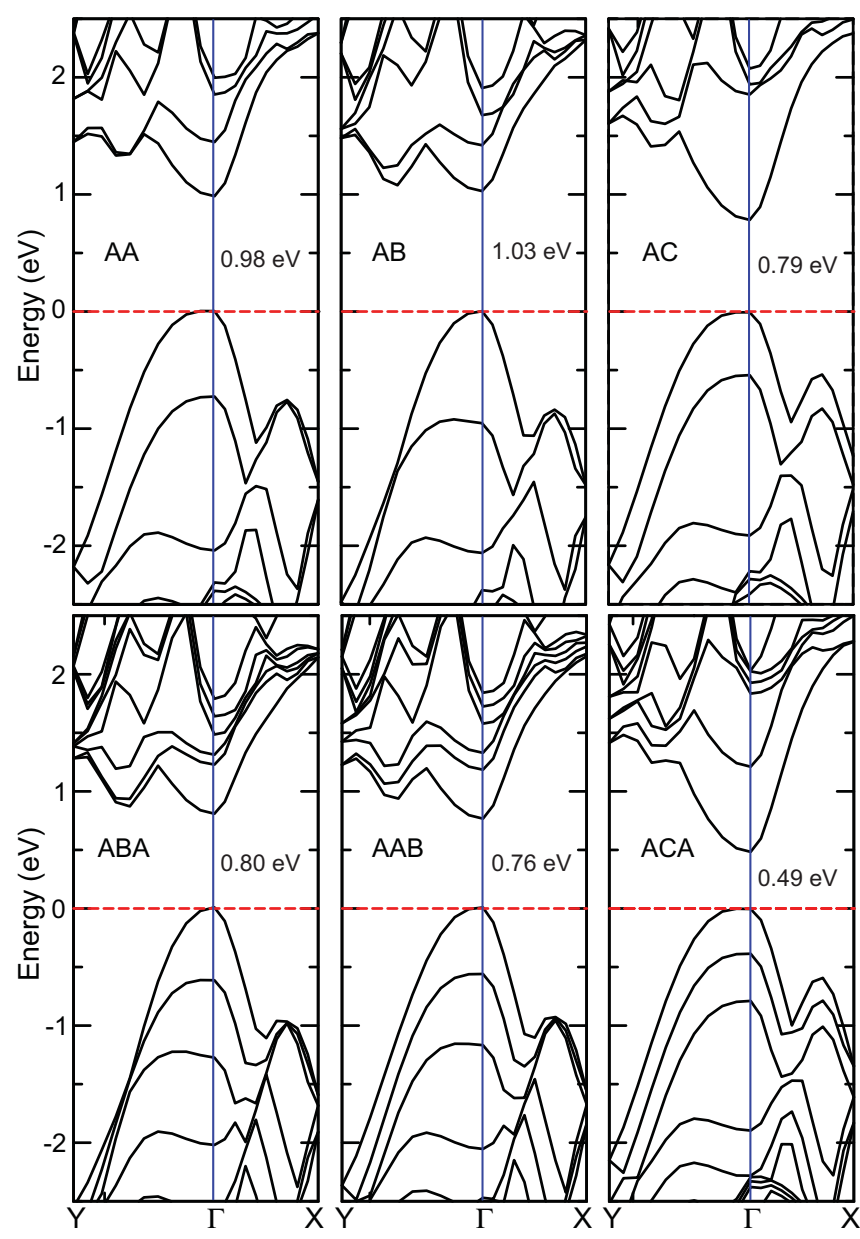

FIG. 3. (Color online) Band structures for different stacking configurations of bilayer (top figures) and trilayer (bottom figures) phosphorene computed with HSE06 along the $Y-\Gamma-X$ direction. The band gap values are also given for each structure. The Fermi level is set at $0 \mathrm{eV}$. 
(a) Two Layers Conduction band

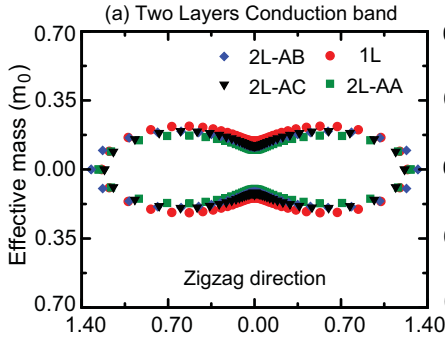

(c) Three Layers Conduction band

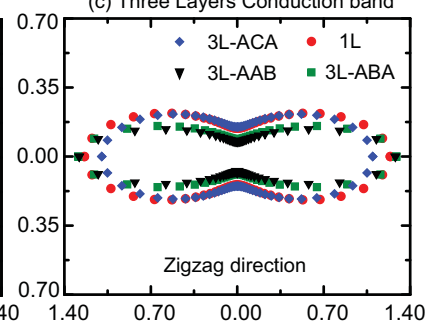

(b) Two Layers Valence band
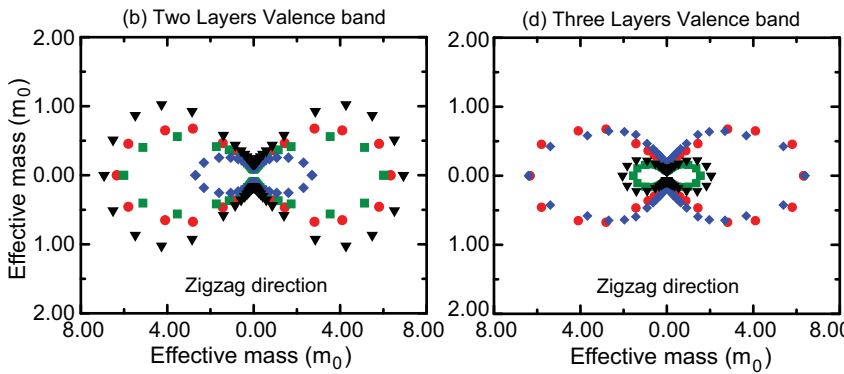

FIG. 4. (Color online) Direction-dependent hole and electron effective masses at the $\Gamma$ point for the different number of layers and different types of stacking. $x(y)$ axis denotes the zigzag (armchair) direction of black phosphorus. Each data point represents the end point of a vector whose amplitude corresponds to the effective mass in units of $m_{0}$ and the direction of this vector corresponds to the direction in $k$ space along which the mass is calculated.

of the band energies with respect to $k_{i}$, we use a dense twodimensional $k$-point grid centered at the $\Gamma$ point. Numerical second derivatives are obtained by using forward and backward differences. Small steps in $k$ space are chosen such that the numerical derivative is converged at each $k$ point. Since the effective mass is directly proportional to the inverse of the curvature of the band dispersion, both electrons and holes have much larger effective masses along the zigzag direction. For different directions, the effective masses can even differ by an order of magnitude. For instance, while the electron effective mass is calculated as $0.10 m_{0}$ along the armchair direction, it becomes $1.26 m_{0}$ along the zigzag direction for $\mathrm{AB}$ stacking of bilayer black phosphorus. Here $m_{0}$ denotes the effective mass of a free electron.

It is worth revealing the dependence of the effective masses on both the number of layers and the stacking type. The hole and electron effective mass tensor for monolayer, bilayer, and trilayer black phosphorus is diagrammatically illustrated in Fig. 4. Each data point represents the end point of a vector [whose origin is at $(0,0)$ in Fig. 4] that shows the effective mass at the $\Gamma$ point calculated along the corresponding reciprocal space direction. Not only the effective masses along the zigzag and armchair directions but also along all other reciprocal space directions between them are shown in Fig. 4. Thus the mass is converted into a vector as follows:

$$
\vec{m}^{*}=\hbar^{2}\left(\frac{\partial^{2} E}{\partial k^{2}}\right)^{-1} \vec{e}_{\vec{k}},
$$

where the derivatives is taken along $\vec{k}=k \vec{e}_{\vec{k}}$.

The first observation is that their angular variation of both electron and hole masses has approximately an " 8 " shape, which indicates clearly the anisotropic nature of the electronic properties of black phosphorus. In addition, the variation of the effective hole mass is quite different as compared to that of the effective electron mass. Consistent with previous work [20], the effective mass of the hole along the zigzag direction decreases with increasing number of layers for the lowest energy stacking types, i.e., $\mathrm{AB}$ and $\mathrm{ABA}$. For the armchair direction, no appreciable variation is observed. However, the type of stacking may have a significant impact on the effective masses. As one changes the stacking from $\mathrm{ABA}$ to $\mathrm{AAB}$ in trilayer black phosphorus, the hole effective mass along the zigzag direction increases from $1.55 m_{0}$ to $6.39 m_{0}$. Such a large change will strongly reduce the mobility of holes along the zigzag direction. These anisotropic electronic properties force confining the carriers in an effective one-dimensional environment along the armchair direction. Since both bilayer and trilayer black phosphorus for $\mathrm{AA}, \mathrm{AC}, \mathrm{AAB}$, and $\mathrm{AAC}$ stackings have a rather flat band structure for the valence band along the zigzag direction, the hole effective mass is quite large for these types of stackings; see Figs. 3 and 4. Note that departing from the lowest energy stacking type, the band structures retain its monolayer behavior for the valence band along the zigzag direction in both bilayer and trilayer black phosphorus. However, the electron effective mass is insensitive to the stacking along the zigzag direction. Interestingly, both electron and hole effective masses along the armchair direction are about an order of magnitude smaller than those along the zigzag direction and are hardly affected by the type of stacking. Our calculations suggest that an appropriate control of the type of stacking may help to control especially the hole conduction in phosphorene-based electronic devices.

Figure 5 shows the band-decomposed charge densities corresponding to the valence band maximum (VBM) and the conduction band minimum (CBM) at the $\Gamma$ point. In all cases, the CBM has a delocalized nature along the zigzag direction of black phosphorus. Stacking especially modifies the CBM in $\mathrm{AC}$ stacking in the bilayer and ACA in the trilayer. We notice partially delocalized states in the interfacial area between layers, which can be associated with a larger band gap lowering in $\mathrm{AC}$ and ACA stacked black phosphorus as compared to other stacking types. The VBM charge distribution is relatively insensitive to the stacking type and has a localized nature. Due to the presence of different interlayer interactions in the trilayer case, the contribution of individual layers to the charge density at the VBM varies. As is clear from Fig. 5 different stacking types result in different $\pi-\pi$ interaction distances and strengths, which are responsible for the tuning of the electronic band gaps.

\section{OPTICAL PROPERTIES}

The optical gaps $\left(E_{\mathrm{opt}}\right)$ and exciton binding energies ( $\left.E_{\text {bind }}=E_{\text {gap }}^{G_{0} W_{0}}-E_{\text {opt }}\right)$ are listed in Table I. Though the stacking types have a small effect on the calculated total energy and lattice parameters, we find that not only $E_{\text {gap }}$ but also $E_{\mathrm{opt}}$ and $E_{\text {bind }}$ are sensitive to the type of stacking. This means that the electronic and optical properties of black phosphorus can be modified by changing the type of stacking [16]. Before discussing the optical properties of bilayer and trilayer black phosphorus, it is informative to summarize the results for the 


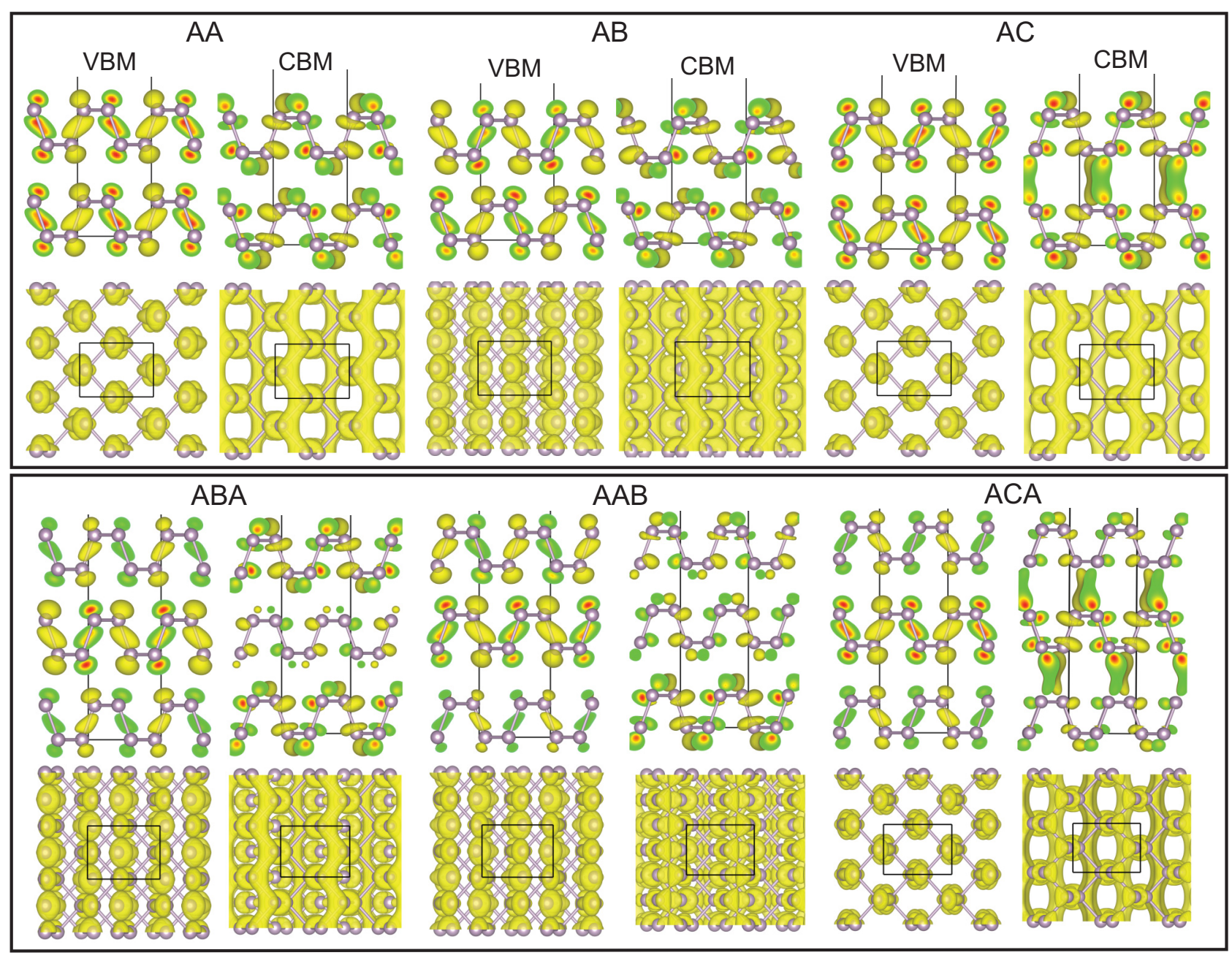

FIG. 5. (Color online) Side and top views of decomposed charge densities corresponding to the VBM and CBM for both bilayer (top figures) and trilayer (bottom figures) black phosphorus at the $\Gamma$ point.

single-layer case. The quasiparticle band gap obtained from $G_{0} W_{0}$ is $2.3 \mathrm{eV}$. In line with our work, photoluminescence excitation spectroscopy finds a quasiparticle band gap of $2.2 \mathrm{eV}$ [55]. Such a large modification of PBE-GGA band gap upon inclusion of many-body effects is a result of the enhanced electron-electron correlation due to confinement effect in 2D black phosphorus. Similarly, the experimental optical gap for single-layer black phosphorus was measured to be around $1.45 \mathrm{eV}$ in Ref. [11] and $1.3 \mathrm{eV}$ in Ref. [55], in agreement with our calculated value of $1.60 \mathrm{eV}$ [29]. Due to the reduced dimensionality and weak screening, single-layer black phosphorus has a large exciton binding energy of $0.7 \mathrm{eV}$, which is in good agreement with recent theoretical works [27,28]. By applying a tensile strain of $4 \%, E_{\text {bind }}$ can be further increased to $0.83 \mathrm{eV}$, placing it among the highest of the 2D materials [29]. The optical properties of single-layer black phosphorus shows a strong orientation dependence such that the wave function of bound excitons is extended along the armchair direction.

Figure 6 displays the optical absorption spectra $\left(\varepsilon_{2}\right)$ of bilayer and trilayer black phosphorus having different stacking types for light polarized along the armchair and zigzag directions. We also show the electronic band gap

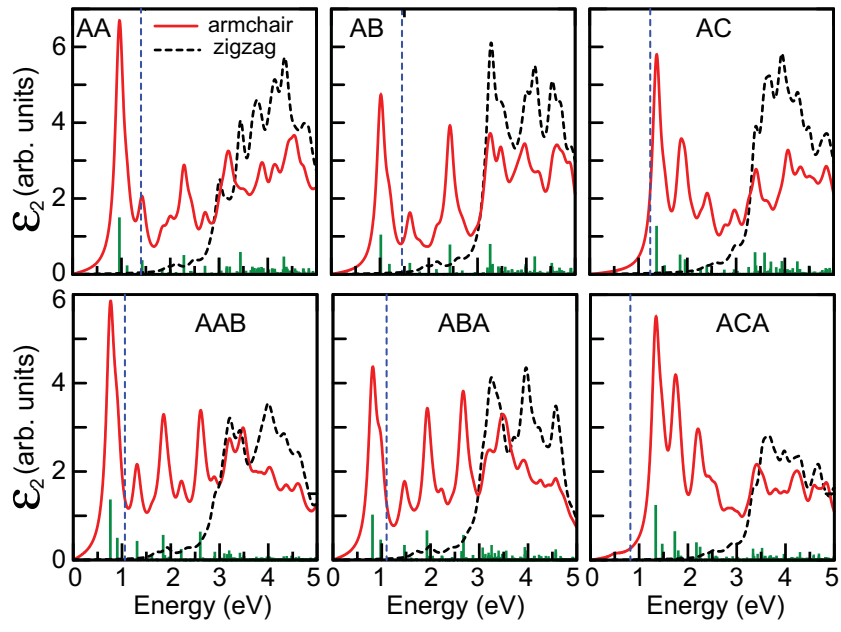

FIG. 6. (Color online) $G_{0} W_{0}+$ BSE absorption spectra for bilayer (top figures) and trilayer (bottom figures) black phosphorus with different stacking types. Blue vertical dashed line mark the electronic band gap calculated at the level of $G_{0} W_{0}$. Green vertical lines represent the relative oscillator strengths for the different optical transitions. 
values calculated with $G_{0} W_{0}$ in the same plots. Similar to single-layer black phosphorus, the optical absorption spectra display a strong orientation dependence. Due to the reduced dimensionality and weak screening, excitonic effects largely shape the optical spectra. A strong absorption peak along the armchair direction is located around $0.96 \mathrm{eV}$ for AA, $1.02 \mathrm{eV}$ for $\mathrm{AB}, 0.81 \mathrm{eV}$ for $\mathrm{ABA}$, and $0.76 \mathrm{eV}$ for $\mathrm{AAB}$, which are bound excitonic states with an $E_{\text {bind }}$ of $0.44 \mathrm{eV}$ for bilayer and $0.30 \mathrm{eV}$ for trilayer phosphorus. All these peaks originate from the interband transition from VBM to CBM. However, AC and ACA stackings behave differently. In spite of their smaller electronic band gaps as compared to other stacking types, we observe a strong absorption peak around $1.37 \mathrm{eV}$ for $\mathrm{AC}$ stacking in the bilayer and $1.34 \mathrm{eV}$ for ACA stacking in the trilayer, which are located $0.13 \mathrm{eV}$ for the former and $0.52 \mathrm{eV}$ for the latter above the quasiparticle band gap. Actually, there is a very small absorption feature at $0.79 \mathrm{eV}$ for $\mathrm{AC}$ stacking and $0.49 \mathrm{eV}$ for ACA stacking with a very small oscillator strength, corresponding to an excitonic state with a binding energy of $0.46(0.33) \mathrm{eV}$ in the former (latter) stacking. These optical transitions result in a very small intensity in a photoluminescence experiment. So, their observation is expected to be difficult. This weak intensity may be linked to the different behavior of the CBM at the $\Gamma$ point. While the formation of AC and ACA are energetically less favorable as compared to other stacking types, the CBM has a delocalized nature in the interfacial region, which further enhances the interlayer interaction, thereby preventing the excitation of a bound exciton. The optical response of bilayer (trilayer) black phosphorus can be tuned from $0.96(0.76) \mathrm{eV}$ for AA (AAB) to 1.37 (1.34) eV for AC (ACA). Such a large change in the optical absorption energy window can be utilized in the design of efficient photovoltaic and optoelectronic devices.

As expected, $E_{\text {bind }}$ decreases with increasing number of stacking layers due to the enhancement of the interlayer interaction. In Ref. [27], $E_{\text {bind }}$ has been found to be only $30 \mathrm{meV}$ for an infinite number of layers, i.e., bulk black phosphorus. We find that the bound exciton wave functions are mainly extended along the armchair direction. Adversely, there is no optically bright exciton whose wave function is extended along the zigzag direction. This means that BP is transparent to polarized light along the zigzag direction and when an exciton is optically excited, it forms along the armchair direction. Due to the selection rules associated with the symmetries of black phosphorus, the prominent absorption peaks along the zigzag direction start at energies about $2 \mathrm{eV}$ above the quasiparticle band gap. These peculiar optical properties make black phosphorus a promising optical linear polarizer, which can be used in several applications, for instance in liquid-crystal displays.

Lastly, we discuss the impact of the interlayer distance on the exciton binding energy. We first checked the impact of the type of van der Waals functional on the lattice parameters and interlayer distances. It was previously shown that the use of different functionals results in different binding energies and interlayer distances in 2D layer materials [56]. If we use a more accurate nonlocal optB88-vdw exchangecorrelation functional [57,58], the lattice parameters and interlayer distances vary by at most $1 \%-2 \%$. In other words, this functional slightly decreases (increases) in-plane lattice parameters (interlayer distances). Increasing the interlayer distance from $3.09 \AA$ to $3.21 \AA$ in $\mathrm{AB}$ stacking changes the electronic gap, optical gap, and exciton binding energy by at most 40 meV. In Ref. [27] where a truncation of long-ranged interactions was employed, the quasiparticle band gap of the $\mathrm{AB}$ stacked phosphorene varies by less than $60 \mathrm{meV}$ when we change the interlayer distance by $0.5 \AA$. Since such truncation scheme is not implemented in the VASP code, the dependence of electronic and optical properties on the amount of vacuum region should be carefully considered $[59,60]$. In a very recent study, the calculated quasiparticle band gap approaches $2.1 \mathrm{eV}$ for $G_{0} W_{0}$ and $2.3 \mathrm{eV}$ for $G_{2} W_{0}$ as the vacuum size approaches infinity, in excellent agreement with our band gap value of $2.3 \mathrm{eV}$ [60]. While the quasiparticle band gap and the exciton binding energy of black phosphorus have a strong dependence on the amount of vacuum size $\left(L_{z}\right)$ used in the calculations, the optical band gap is found to be well converged with $L_{z}>10 \AA$ [60]. We here used $L_{z}>$ $15 \AA$, which is sufficient to get converged optical gaps. It was previously shown that the compressive strain (i.e., reducing in-plane lattice parameters) tends to decrease the electronic gap, optical gap, and exciton binding energy of monolayer black phosphorus [29]. This means that since the use of more accurate van der Waals functionals (for instance optB88$\mathrm{vdw}$ ) predicts much smaller in-plane lattice parameters, the calculated quasiparticle band gap, optical gap, and exciton binding energy are found to decrease if we employ these nonlocal exchange-correlation functionals. We notice here that the opposite effect of optB88-vdw exchange-correlation functional on in-plane lattice parameters and interlayer separations partially results in a cancellation of computational errors which is inherent in $G W$ and BSE calculations. While $\mathrm{AA}$ and $\mathrm{AB}$ stackings have quite different interlayer distances, $E_{\text {bind }}$ is found to be around $0.44 \mathrm{eV}$ for both types of stacking. Similarly, in the trilayer case, in spite of different interlayer separations in ABA and AAB stackings, $E_{\text {bind }}$ converges to an average value of $0.30 \mathrm{eV}$. Due to the vacuum surrounding these stacked layers, $E_{\text {bind }}$ hardly changes with interlayer separation.

\section{CONCLUSION}

In summary, our results show that the number of layers and the type of stacking have a significant impact on the electronic and optical properties of black phosphorus. The change of the band gap with stacking is directly related with the upward (downward) shift of the VBM (CBM), originating from the presence of different interlayer interactions. However, the nature of the band gap (i.e., direct band gap at the $\Gamma$ point) is insensitive to the number of layers and the type of stacking. The hole effective mass strongly depends on the type of stacking. While the hole effective mass at the $\Gamma$ point along the zigzag direction decreases with increasing number of layers, the deviation from the lowest energy structures results in quite flat valence bands, thereby increasing the hole effective masses along the zigzag direction.

We find that the absorption energy window of black phosphorus can be tuned by changing the number of layers and by the type of stacking. While stacking alters the optical 
gap of bilayer black phosphorus up to $0.4 \mathrm{eV}$, it may result in a $0.6 \mathrm{eV}$ change in the trilayer case.

Previously, strain engineering has been shown to modify the electronic and optic properties of black phosphorus [29]. Here, we show that playing with the number of layers and the type of stacking appears as an exciting alternative way to tune the optical response and the electrical properties of black phosphorus. Finally, it is worth mentioning that the anisotropic optical and electronic properties can be utilized to distinguish the type of stacking and the orientations of few-layer black phosphorous in experiments.

\section{ACKNOWLEDGMENTS}

This work was supported by the Flemish Science Foundation (FWO-Vl) and the Methusalem foundation of the Flemish government. Computational resources were provided by TUBITAK ULAKBIM, High Performance and Grid Computing Center (TR-Grid e-Infrastructure), and HPC infrastructure of the University of Antwerp (CalcUA) a division of the Flemish Supercomputer Center (VSC), which is funded by the Hercules foundation. C.S. acknowledges support from Turkish Academy of Sciences (TUBA-GEBIP).
[1] K. S. Novoselov, A. K. Geim, S. V. Morozov, D. Jiang, Y. Zhang, S. V. Dubonos, I. V. Grigorieva, and A. A. Firsov, Science 306, 666 (2004).

[2] K. S. Novoselov, A. K. Geim, S. V. Morozov, D. Jiang, M. I. Katsnelson, I. V. Grigorieva, S. V. Dubonos, and A. A. Firsov, Nature (London) 438, 197 (2005).

[3] L. Liao, Y.-C. Lin, M. Bao, R. Cheng, J. Bai, Y. Liu, Y. Qu, K. L. Wang, Y. Huang, and X. Duan, Nature (London) 467, 305 (2010).

[4] F. Schwierz, Nat. Nanotechnol. 5, 487 (2010).

[5] Y. Wu, Y.-M. Lin, A. A. Bol, K. A. Jenkins, F. Xia, D. B. Farmer, Y. Zhu, and P. Avouris, Nature (London) 472, 74 (2011).

[6] K. F. Mak, C. Lee, J. Hone, J. Shan, and T. F. Heinz, Phys. Rev. Lett. 105, 136805 (2010).

[7] B. Radisavljevic, A. Radenovic, J. Brivio, V. Giacometti, and A. Kis, Nat. Nanotechnol. 6, 147 (2011).

[8] Y. Yoon, K. Ganapathi, and S. Salahuddin, Nano Lett. 11, 3768 (2011).

[9] M. S. Fuhrer and J. Hone, Nat. Nanotechnol. 8, 146 (2013).

[10] L. Li, Y. Yu, G. J. Ye, Q. Ge, X. Ou, H. Wu, D. Feng, X. H. Chen, and Y. Zhang, Nat. Nanotechnol. 9, 372 (2014).

[11] H. Liu, A. T. Neal, Z. Zhu, Z. Luo, X. Xu, D. Tománek, and P. D. Ye, ACS Nano 8, 4033 (2014).

[12] E. S. Reich, Nature (London) 506, 19 (2014).

[13] H. Liu, Y. Du, Y. Deng, and P. D. Ye, Chem. Soc. Rev. 44, 2732 (2015).

[14] L. Kou, T. Frauenheim, and C. Chen, J. Phys. Chem. Lett. 5, 2675 (2014).

[15] M. Buscema, D. J. Groenendijk, G. A. Steele, H. S. J. van der Zant, and A. Castellanos-Gomez, Nat. Commun. 5, 4651 (2014).

[16] J. Dai and X. C. Zeng, J. Phys. Chem. Lett. 5, 1289 (2014).

[17] M. Buscema, D. J. Groenendijk, S. I. Blanter, G. A. Steele, H. S. J. van der Zant, and A. Castellanos-Gomez, Nano Lett. 14, 3347 (2014).

[18] F. Xia, H. Wang, and Y. Jia, Nat. Commun. 5, 4458 (2014).

[19] A. Castellanos-Gomez, L. Vicarelli, E. Prada, J. O. Island, K. L. Narasimha-Acharya, S. I. Blanter, D. J. Groenendijk, M. Buscema, G. A. Steele, J. V. Alvarez, H. W. Zandbergen, J. J. Palacios, and H. S. J. van der Zant, 2D Mater. 1, 025001 (2014).

[20] J. Qiao, X. Kong, Z.-X. Hu, F. Yang, and W. Ji, Nat. Commun. 5, 4475 (2014).

[21] A. S. Rodin, A. Carvalho, and A. H. Castro Neto, Phys. Rev. Lett. 112, 176801 (2014).

[22] R. Fei and L. Yang, Nano Lett. 14, 2884 (2014).
[23] S. Appalakondaiah, G. Vaitheeswaran, S. Lebegue, N. E. Christensen, and A. Svane, Phys. Rev. B 86, 035105 (2012).

[24] Z. Zhu and D. Tománek, Phys. Rev. Lett. 112, 176802 (2014).

[25] J. Guan, Z. Zhu, and D. Tománek, Phys. Rev. Lett. 113, 046804 (2014).

[26] Y. Aierken, D. Çakır, C. Sevik, and F. M. Peeters, Phys. Rev. B 92, 081408 (2015).

[27] V. Tran, R. Soklaski, Y. Liang, and L. Yang, Phys. Rev. B 89, 235319 (2014).

[28] A. S. Rodin, A. Carvalho, and A. H. Castro Neto, Phys. Rev. B 90, 075429 (2014)

[29] D. Çakır, H. Sahin, and F. M. Peeters, Phys. Rev. B 90, 205421 (2014).

[30] A. Chaves, T. Low, P. Avouris, D. Çakır, and F. M. Peeters, Phys. Rev. B 91, 155311 (2015).

[31] L. Seixas, A. S. Rodin, A. Carvalho, and A. H. Castro Neto, Phys. Rev. B 91, 115437 (2015).

[32] M. Mehboudi, K. Utt, H. Terrones, E. O. Harriss, A. A. Pacheco SanJuan, and S. Barraza-Lopez, Proc. Natl. Acad. Sci. USA 112, 5888 (2015).

[33] G. Kresse and J. Furthmüller, Comput. Mater. Sci. 6, 15 (1996).

[34] G. Kresse and J. Furthmüller, Phys. Rev. B 54, 11169 (1996).

[35] J. P. Perdew, K. Burke, and M. Ernzerhof, Phys. Rev. Lett. 77, 3865 (1996).

[36] J. Heyd, G. E. Scuseria, and M. Ernzerhof, J. Chem. Phys. 118 8207 (2003).

[37] J. Paier, M. Marsman, K. Hummer, G. Kresse, I. C. Gerber, and J. G. Ángyán, J. Chem. Phys. 125, 249901 (2006).

[38] J. Heyd, G. E. Scuseria, and M. Ernzerhof, J. Chem. Phys. 124, 219906 (2006).

[39] P. E. Blöchl, Phys. Rev. B 50, 17953 (1994).

[40] H. J. Monkhorst and J. D. Pack, Phys. Rev. B 13, 5188 (1976).

[41] S. Grimme, J. Comput. Chem. 27, 1787 (2006).

[42] D. Y. Qiu, F. H. da Jornada, and S. G. Louie, Phys. Rev. Lett. 111, 216805 (2013).

[43] H.-P. Komsa and A. V. Krasheninnikov, Phys. Rev. B 86, 241201 (2012).

[44] H.-P. Komsa and A. V. Krasheninnikov, Phys. Rev. B 88, 085318 (2013).

[45] A. Ramasubramaniam, Phys. Rev. B 86, 115409 (2012).

[46] E. E. Salpeter and H. A. Bethe, Phys. Rev. 84, 1232 (1951).

[47] G. Onida, L. Reining, and A. Rubio, Rev. Mod. Phys. 74, 601 (2002).

[48] L. Hedin, Phys. Rev. 139, A796 (1965).

[49] M. S. Hybertsen and S. G. Louie, Phys. Rev. B 34, 5390 (1986) 
[50] R. W. Godby, M. Schlüter, and L. J. Sham, Phys. Rev. B 37, 10159 (1988).

[51] M. Shishkin and G. Kresse, Phys. Rev. B 74, 035101 (2006).

[52] M. Shishkin and G. Kresse, Phys. Rev. B 75, 235102 (2007).

[53] M. Shishkin, M. Marsman, and G. Kresse, Phys. Rev. Lett. 99, 246403 (2007).

[54] J. Paier, M. Marsman, and G. Kresse, Phys. Rev. B 78, 121201 (2008).

[55] X. Wang, A. M. Jones, K. L. Seyler, V. Tran, Y. Jia, H. Zhao, H. Wang, L. Yang, X. Xu, and F. Xia, Nat. Nanotechnol. 10, 517 (2015).
[56] T. Björkman, A. Gulans, A. V. Krasheninnikov, and R. M. Nieminen, Phys. Rev. Lett. 108, 235502 (2012).

[57] M. Dion, H. Rydberg, E. Schröder, D. C. Langreth, and B. I. Lundqvist, Phys. Rev. Lett. 92, 246401 (2004).

[58] J. Klimeš, D. R. Bowler, and A. Michaelides, J. Phys.: Condens. Matter 22, 022201 (2010).

[59] L. Wirtz, A. Marini, and A. Rubio, Phys. Rev. Lett. 96, 126104 (2006).

[60] J.-H. Choi, P. Cui, H. Lan, and Z. Zhang, Phys. Rev. Lett. 115, 066403 (2015). 